\title{
Analysis of the Risks of Public Transportation, Escalators: A Case Study of Children Falling off the Escalators
}

\author{
Aiguo Liu ${ }^{1,2}$, Jun $\mathrm{Ou}^{1}{ }^{1, *}$, Chen $\mathrm{Xu}^{1}$, Weimin $\mathrm{Xiao}^{3}$, CHangjie $\mathrm{Li}^{1}$, Yanbing $\mathrm{Li}^{1}$, Sen $\mathrm{Jia}^{1,2}$ \\ ${ }^{1}$ Henan Institute of Special Equipment Safety Inspection and Testing, Zhengzhou 450016, China \\ ${ }^{2}$ National Center for Quality Supervision and Inspection of Overhead Gantry Cranes and \\ Lightweight Hoists, Xinxiang 453400, China \\ ${ }^{3}$ Shenzhen Academy of Metrology and Quality Inspection, Shenzhen 518109, China
}

\section{城市公共交通自动扶梯风险分析：以自动扶梯儿 童芏落事故 ATA 应用为例}

\author{
刘爱国 ${ }^{1 、 2}$, 欧俊 ${ }^{1, *}$, 许晨 ${ }^{1}$, 肖伟敏 ${ }^{3}$, 李昌杰 ${ }^{1}$, 李雁冰 ${ }^{1}$, 贾森 ${ }^{1 、 2}$ \\ ${ }^{1}$ 河南省特种设备安全检测研究院, 郑州 450016, 中国 \\ ${ }^{2}$ 国家桥架类及轻小型起重机械质量监督检验中心, 新乡 453400 , 中国 \\ ${ }^{3}$ 深圳市计量质量检测研究院, 深圳 518109, 中国
}

\begin{abstract}
In view of urban public traffic safety, the accident tree analysis(ATA) theory was applied to construct an accident tree model which taken the children fall accident in the escalator area as the top event. According to the accident tree model, the Boolean algebraic function expressions of the accident tree and the success tree were obtained, the minimum cut set and the minimum path set were also determined, and the ranking of structural importance of the basic events was obtained. According to the similar events' probability of relevant literatures, the basic events' probability were initially assigned, the ranking of probability importance and critical importance of each basic event structure were also obtained. Through the risk assessment and analysis of the results of the three important rankings, it was shown that children climbing the handrails,
\end{abstract}

no supervision, inadequate adult supervision, no pre-fall prevention devices, post-fall prevention devices were the important factors affecting the children fall accident.

Keywords: public transportation; escalators; fall accident; Accident Tree Analysis(ATA); the calculation of important degree; risk analysis

摘要

针对城市公共交通安全, 应用事故树 分析 (ATA) 理论, 构建了以自动扶梯相邻 区域儿童意外坠落伤害事故为顶上事件的 事故树模型。由事故树模型, 得到事故树 和成功树的布尔代数函数表达式, 求出基 本事件的最小割集和最小径集, 得出基本 事件的结构重要度排序。参照相关文献类 似事件的概率, 对本基本事件的概率进行 了初始赋值, 并得到了基本事件的概率重 要度和临界重要度排序。对三个重要度系

*通讯作者 E-mail： ouy6666@126. com。

作者简介: 刘爱国 (1959-), 男, 河南郑州人, 正高级工程师, 主要研究方向为特种设备风险分析与控制、型式试验 和检测检验技术管理。 
数排序结果的风险评估, 分析表明儿童攀 爬扶手带、无人监护、家长监护不到位、 未设坠后防护装置以及未设坠前阻挡装置 等, 是导致儿童意外坠落事故发生的重要 原因。

关键词: 公共交通; 自动扶梯; 坠落事故; 事故树分析（ATA）; 重要度计算; 风险分 析

\section{1. 引言}

公交型自动扶梯在火车站、地铁、机 场等城市交通枢纽中得到广泛应用, 以每 天满载、持续频繁地运送乘客是其主要特 点之一。截止 2017 年底, 我国自动扶梯和 自动人行道总量已达到 84 余万台。随着使 用数量增加, 高处坠落、夹伤、跌（滑） 倒等事故日渐凸显。其中, 自动扶梯坠落 事故占乘客伤害事故总数的 $11 \%{ }^{[1]}$, 且受伤 害的主要对象为儿童群体。如何防范儿童 在自动扶梯上意外坠落, 引起了社会的广 泛关注, 已成为城市公共安全的重要任务 之一。

在自动扶梯安全性评估和乘客乘用风 险防范方面, 国内外专家学者都做了大量 的研究工作, Bardyshev 0、Popov V 等人 ${ }^{[2]}$ 对自动扶梯超过服务期限后, 以便可能延 长其服务年限, 提出了应用经验和对技术 状况进行评估的新方法; 蔡志明、邵辉 ${ }^{[3]}$ 采 用系统动力学的方法, 并利用 VENSIM 软件 建立的风险概率模型, 对乘客风险概率与 自动扶梯安全性进行模拟和计算, 提出了 乘坐自动扶梯的风险管理措施与建议等。 这些研究对于提高自动扶梯本质安全、健 全使用场所警示标识, 以及约束乘客遵守 乘用规则等方面起到了很重要的指导作用。 但对自动扶梯相邻区域危险空间和与建筑 物间存在的空隙等风险识别研究比较贵乏。 本文应用 ATA 方法, 对自动扶梯相邻区域 的风险进行了识别与分析, 为儿童意外坠 落伤害风险防范提供可借鉴的理论依据。

\section{2. 茎落伤害风险事故树模型}

\section{1 事故树分析法简介}

事故树分析法 (ATA) 起源于故障树分
析法 (FTA)。即由顶上事件 (发生的事故) 开始, 然后分析其中间事件, 最后找出基 本事件或底事件（事故的基本原因或最初 原因)。事故树分析法在产品质量、系统工 程以及对事故的定性、定量和事故发生的 潜在原因以及因果关系等方面 ${ }^{[4] ~[6]}$ 得到较 为广泛的应用。

\section{2 事故树模型建立}

首先确定导致儿童意外芏落伤害事故 的顶上事件 $T$, 顶上事件 $T$ 即为儿童意外坠 落伤害。通过从 “人、机、环” 三要素进 行综合分析判断, 确定了导致顶上事件发 生的 12 个中间事件 MI M12 和 19 个基本 事件 X1 X19 (见表 1、表 2)。建立了儿童 意外坠落伤害风险事故树模型, 如图 1 所

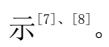

\begin{tabular}{cc} 
& 表 1 中间事件 \\
\hline 符号 & 中间事件 \\
\hline$M 1$ & 儿童的意外坠落事件 \\
$M 2$ & 坠落后防护设施缺陷 \\
$M 3$ & 儿童的坠落倾向明显 \\
$M 4$ & 坠落前阻挡设施缺陷 \\
$M 5$ & 坠落后无防护措施 \\
$M 6$ & 现场监护和监控缺失 \\
$M 7$ & 儿童攀爬扶手带等危险动作 \\
$M 8$ & 无坠落前阻挡装置 \\
$M 9$ & 荫落前阻挡措施不当 \\
$M 10$ & 安全管理缺陷 \\
$M 11$ & 现场监控设备缺陷 \\
$M 12$ & 使用监管方面缺失 \\
\hline
\end{tabular}

\section{3 基本事件的最小割集}

最小割集在事故树中反映了系统的危 险性, 任意一个最小割集发生都会导致顶 上事故的发生 ${ }^{[9]}$ 。

$$
\begin{gathered}
\text { 布尔代数法函数表达式为: } \\
T=M 1 M 2=M 3 M 4(X 1+X 2+M 5)= \\
(M 6 M 7)(M 8+M 9+X 3) \\
(X 1+X 2+X 4+X 5+X 6+M 10) \\
=(X 7+X 8+M 11)(X 9+X 10) \\
(X 11+X 12+M 10+X 6+M 12+X 13+X 3) \\
(X 1+X 2+X 4+X 5+X 6+X 14+X 15)=
\end{gathered}
$$




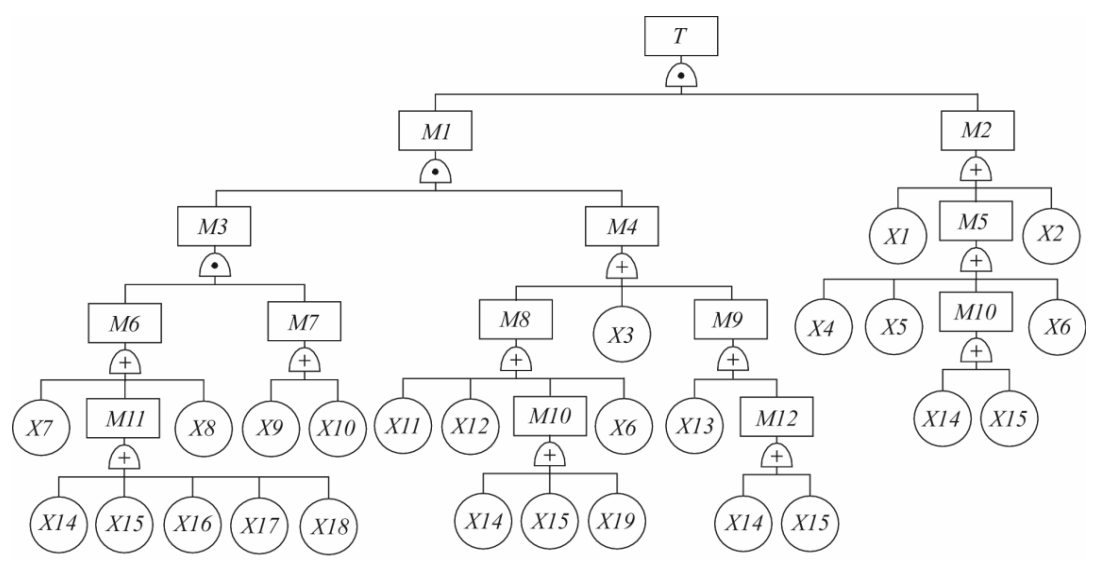

图 1 坠落伤害风险事故树模型

得出最小割集共 214 项（略), 每一项 最小割集代表一种事故产生模式, 说明顶 上事件发生的潜在危险因素（或组合）的 可能性有 214 种情况。

\section{4 基本事件的最小径集}

最小径集表示了系统的安全性。把图 1 中事件发生用事件不发生代替, 把 “与门” 用 “或门” 代替、“或门” 用 “与门” 代替, 基本事件变为 “非”, 便得到了与原事故树 对偶的成功树。对成功树求最小割集, 便 得到原事故树的最小径集。用 $T^{\prime}$ 表示顶上 事件不发生, 则成功树的布尔代数表达式 为:

$T^{\prime}=M 1^{\prime}+M 2^{\prime}=M 3^{\prime}+M 4^{\prime}+X 1^{\prime} X 2^{\prime} M 5^{\prime}=$ $M 6^{\prime} M 7^{\prime}+M 8^{\prime} M 9^{\prime} X 3^{\prime}+X 1^{\prime} X 2^{\prime} X 4^{\prime} X 5^{\prime} X 6^{\prime} M 10^{\prime}$ $=X 7^{\prime} X 8^{\prime} M 11^{\prime}+X 9^{\prime} X 10^{\prime}$ $+X 11^{\prime} X 12^{\prime} M 10^{\prime} X 6^{\prime} M 12^{\prime} X 13^{\prime} X 3^{\prime}$ $+X 1^{\prime} X 2^{\prime} X 4^{\prime} X 5^{\prime} X 6^{\prime} X 14^{\prime} X 15^{\prime}$

$=X 7^{\prime} X 8^{\prime} X 16^{\prime} X 17^{\prime} X 18^{\prime} X 14^{\prime} X 15^{\prime}+X 9^{\prime} X 10^{\prime}$

$+X 11^{\prime} X 12^{\prime} X 14^{\prime} X 15^{\prime} X 19^{\prime} X 6^{\prime} X 14^{\prime} X 13^{\prime} X 3^{\prime}$ $+X 1^{\prime} X 2^{\prime} X 4^{\prime} X 5^{\prime} X 6^{\prime} X 14^{\prime} X 15^{\prime}$

得到本事故树的四个最小径集, 分别 为:

$$
P_{l}=\{X 1, X 2, X 4, X 5, X 6, X 14, X 15\} ;
$$
$P_{2}=\{X 3, X 6, X 11, X 12, X 13, X 14, X 15, X 19\} ;$ $P_{3}=\{X 7, X 8, X 14, X 15, X 16, X 17, X 18\}$; $P_{4}=\{X 9, X 10\}$ 。
3. 事故树风险分析

\section{1 基本事件的结构重要度}

确定基本事件结构重要度顺序可应用近 似公式 (1) 计算。

$$
I_{\phi}(i)=\sum_{X_{i} \in k_{i}} \frac{1}{2^{n_{i}-1}}
$$

式中: $I_{\varphi}(i)$-一第 $i$ 个基本事件结 构重要度 $I_{\varphi}(i)$ 的近似判别值, $I_{\varphi}(i)$ 值大者, 则重要度大;

$X_{i} \in K_{i}$ 一一基本事件 $x_{i}$ 属于 $K_{i}$ 最小割 (径) 集;

$n_{i}$ 一一第 $i$ 个基本事件所在的第 $K_{i}$ 最 小割 (径) 集中包含的基本事件总数。

各基本事件结构重要度的结果分别为: $I_{\Phi}(X 1)=I_{\Phi}(X 2)=I_{\Phi}(X 4)=I_{\Phi}(X 5)=I_{\Phi}(X 7)=$ $I_{\Phi}(X 8)=I_{\Phi}(X 16)=I_{\Phi}(X 17)=I_{\Phi}(X 18)=1 / 64$; ${ }_{\phi}(X 3)=I_{\Phi}(X 11)=I_{\Phi}(X 12)=I_{\phi}(X 13)=$ $I_{\phi}(X 19)=1 / 128 ; \quad I_{\phi}(X 6)=3 / 128 ;$

$I_{\phi}(X 14)=I_{\Phi}(X 15)=5 / 128 ;$

$I_{\varphi}(X 9)=I_{\phi}(X 10)=1 / 2$ 。

各基本事件结构重要度的排序为:

$I_{\phi}(X 9)=I_{\Phi}(X 10)>I_{\phi}(X 14)$

$=I_{\Phi}(X 15)>I_{\Phi}(X 6)>I_{\Phi}(X 1)=I_{\Phi}(X 2)$

$=I_{\Phi}(X 4)=I_{\Phi}(X 5)=I_{\Phi}(X 7)=I_{\phi}(X 8)$

$=I_{\phi}(X 16)=I_{\phi}(X 17)=I_{\phi}(X 18)>I_{\phi}(X 3)$

$=I_{\phi}(X 11)=I_{\Phi}(X 12)=I_{\phi}(X 13)=I_{\phi}(X 19)$ 。 


\section{2 基本事件的概率重要度}

由于顶上事件发生概率函数是 $n$ 个基 本事件发生概率的多重线性函数, 通过对 自变量 $q_{i}$ 求一次偏导, 即可得到该基本事 件的概率重要度系数 $I_{g}(i)$, 由式 (2) 计 算 ${ }^{[17]}$ 求得:

$$
I_{g}(i)=\frac{\partial g}{\partial q_{i}}(i=1,2, \cdots, n)
$$

式中: $I_{g}(i)$ 一一第 $i$ 个基本事件概 率重要度系数;

$$
\begin{aligned}
& g \text {-一顶上事件发生的概率; } \\
& q_{i} \text { 一一第 } i \text { 个基本事件发生的概率。 }
\end{aligned}
$$

\subsection{1 基本事件概率的初始赋值}

由于部分基本事件尚未有公开的统计试 验数据, 分析对比文献 $[7] 、[10] \sim[14]$ 等 相关资料。按照和参考上述文献中相类似 事件的概率, 对本例各基本事件概率进行 了取值, 确定了部分基本事件的概率初始 赋值, 如表 2 所示。

\subsection{2 顶上事件发生的概率计算}

事故树中逻辑或门和最小割集数目较多, 而最小径集数目较少时, 可利用最小径集 来计算顶上事件发生的概率较为方便 ${ }^{[15]}$ 。 本例最小径集有重复事件, 顶上事件发生 的概率按 (3) 式 ${ }^{[16]}$ 计算。

$$
\begin{aligned}
& g=1-\sum_{r=1}^{l} \prod_{x i \in p r}\left(1-q_{\mathrm{i}}\right)+\sum_{1 \leq r \leq s \leq l} \prod_{x i \in p r \cup p s}\left(1-q_{i}\right)^{-} \\
& \sum_{1 \leq r \leq s \leq t \leq l} \prod_{\substack{i \in \in \\
p_{r} \cup p s \cup p_{t}}}\left(1-q_{i}\right)+\cdots+(-1) \prod_{\substack{r=1 \\
x i \in p_{r}}}^{l}\left(1-q_{i}\right) \quad(3) \\
& \text { 式中: }\left.\sum_{r=1}\right|_{x i \in P_{r}}\left(1-q_{i}\right) \text { 一一属于每个不同 }
\end{aligned}
$$

最小径集基本事件不发生的概率之积的代 数和;

1一一最小径集数;

$\left(1-q_{i}\right)$ 一一第 $i$ 个基本事件不发生的 概率;

$$
\sum_{1 \leq r \leq s \leq 1} \prod_{x i \in P_{r} \cup P_{s}} \text { 一一属于任意两个不同最 }
$$

小径集的并事件中的基本事件不发生概率 之积的代数和;

$$
P_{r} 、 P_{s} 、 P_{t} \text { 一一最小径集 }(r 、 s 、 t=1,
$$

$2, \cdots \cdots k)$;

$r 、 s-1$ 最小径集数序数, $r<s<t<1$;

i一一基本事件序数;

$x_{i} \in P r$ 一属于第 $r$ 个最小径集的第 $i$

个基本事件;

$1 \leqslant r<s \leqslant 1$ 一一任意两个最小径集的 组合顺序;

$x_{i} \in P r \cup P_{S}$ 一一属于第 $r$ 个或属于第 $S$ 个最小径集的第 $i$ 个基本事件。

表 2 基本事件概率

\begin{tabular}{cccc}
\hline 符号 & 基本事件 & $q_{i}$ & $1-q_{i}$ \\
\hline$X 1$ & 坠后防护装置损坏 & 0.1 & 0.9 \\
$X 2$ & 坠后防护措施不当 & 0.1 & 0.9 \\
$X 3$ & 坠前阻挡装置损坏 & 0.1 & 0.9 \\
$X 4$ & 未设坠后防护装置 & 0.7 & 0.3 \\
$X 5$ & 坠后防护装置未设计 & 0.006 & 0.994 \\
$X 6$ & 安全资金投入不足 & 0.1 & 0.9 \\
$X 7$ & 无人监护 & 0.1 & 0.9 \\
$X 8$ & 家长监护不到位 & 0.1 & 0.9 \\
$X 9$ & 儿童安全教育不足 & 0.006 & 0.994 \\
$X 10$ & 儿童攀爬扶手带 & 0.7 & 0.3 \\
$X 11$ & 未设前前阻挡装置 & 0.7 & 0.3 \\
$X 12$ & 坠前阻挡装置未设计 & 0.006 & 0.994 \\
$X 13$ & 坠前阻挡方法不当 & 0.1 & 0.9 \\
$X 14$ & 安全管理缺陷 & 0.1 & 0.9 \\
$X 15$ & 主管责任人监管欠缺 & 0.006 & 0.994 \\
$X 16$ & 未装监控设备 & 0.1 & 0.9 \\
$X 17$ & 监控设备缺陷 & 0.01 & 0.99 \\
$X 18$ & 现场监管缺失 & 0.1 & 0.9 \\
$X 19$ & 制造商未告知风险 & 0.003 & 0.997 \\
\hline \multicolumn{3}{c}{ 本例事故树中虽然最小径集较少, 但其 }
\end{tabular}
最小径集的基本事件较多, 利用 (3) 式求 解相当繁琐。通常采取近似计算法, 利用 最小径集逼近法, 只需计算顶上事件发生 概率的近似上限与下限 ${ }^{[17]}$ 范围即可。

$$
\begin{gathered}
\text { 令 } S_{1}=\sum_{r=1}^{l} \prod_{x_{i} \in p_{r}}\left(1-q_{i}\right) ; S_{2}= \\
\sum_{1 \leq r \leq s \leq 1} \prod_{x_{i} \in P_{r} \cup P_{s}}\left(1-q_{i}\right) ; S_{3}=\prod_{\substack{r=1 \\
x_{i} \in P_{r}}}^{N_{p}}\left(1-q_{i}\right)
\end{gathered}
$$

则 $1-S_{1}+S_{2}-S_{3} \leq g \leq 1-S_{1}+S_{2} \quad$ (4)

按 (4) 式取前三项进行计算, 便可得 到较理想的近似值, 以 2.4 给出的事故树 中四个最小径集, 用 (4) 式展开, 消去概 率积中基本事件 $x_{i}$ 不发生概率 $\left(1-q_{i}\right)$ 的重 复事件, 然后将表 2 中各基本事件的不发 
生概率 $\left(1-q_{i}\right)$ 取值代入其展开式, 则顶上 事件发生概率为:

$$
0.3205363 \leqslant g \leqslant 0.3296075
$$

按 $g=1-S_{1}+S_{2}-S_{3}$ 取值, 则:

$$
g=0.3205363 \text { 。 }
$$

\subsection{3 各基本事件概率重要度系数计算}

根据（2）式用最小径集, 计算基本事 件概率重要度系数 $I g(1) \sim \operatorname{Ig}(19)$ 如下:

$$
\begin{aligned}
& I_{g}(1)=\frac{\partial g}{\partial q_{1}}=\left(1-q_{2}\right)\left(1-q_{4}\right)\left(1-q_{5}\right) \\
& \left(1-q_{6}\right)\left(1-q_{14}\right)\left(1-q_{15}\right)\left[1-\left(1-q_{3}\right)\left(1-q_{6}\right)\right. \\
& \left(1-q_{11}\right)\left(1-q_{12}\right)\left(1-q_{13}\right)\left(1-q_{14}\right)\left(1-q_{15}\right) \\
& \left.\left(1-q_{19}\right)\right]\left[1-\left(1-q_{7}\right)\left(1-q_{8}\right)\left(1-q_{14}\right)\left(1-q_{15}\right)\right. \\
& \left.\left(1-q_{16}\right)\left(1-q_{17}\right)\left(1-q_{18}\right)\right]\left[1-\left(1-q_{9}\right)\left(1-q_{10}\right)\right]
\end{aligned}
$$$$
\text { 将表 } 2 \text { 中各基本事件的概率代入上式 }
$$

得: $I_{g}(1) \approx 0.05151996$

同理: $I_{g}(2) \approx 0.05151996 ; I_{g}(3)=$ $I_{g}(13) \approx 0.06998663 ; \quad I_{g}(4) \approx 0.15363252$; $I_{g}(5) \approx 0.04636797 ; \quad I_{g}(6) \approx 0.01368626$; $I_{g}(7)=I_{g}(8)=I_{g}(16)=I_{g}(18) \approx 0.18996297$; $I_{g}(9) \approx 0.08160696 ; \quad I_{g}(10) \approx 0.27039105$; $I_{g}(11) \approx 0.15398467 ; \quad I_{g}(12) \approx 0.0461954$; $I_{g}(14) \approx 0.02109324 ; I_{g}(15) \approx 0.01565711$; $I_{g}(17) \approx 0.17269361 ; I_{g}(19) \approx 0.04608975$ 。

得到各基本事件概率重要度系数的排序 为: $I_{g}(10)>I_{g}(7)=I_{g}(8)=I_{g}(16)=I_{g}(18)>$ $I_{g}(17)>I_{g}(11)>I_{g}(4)>I_{g}(9)>I_{g}(3)=I_{g}(13)>$ $I_{g}(2)=I_{g}(1)>I_{g}(5)>I_{g}(12)>I_{g}(19)>I_{g}(14)>$ $I_{g}(15)>I_{g}(6)$

\section{3 基本事件临界重要度}

\subsection{1 临界重要度}

临界重要度比结构重要度系数和概率 重要度系数, 更能准确反映基本事件对顶 上事件的影响程度。其表达式为:

$$
I_{G}(i)=\frac{\partial \ln g}{\partial \ln q_{i}}=\frac{\partial g}{g} / \frac{\partial q_{i}}{q_{i}}=\frac{q_{i}}{g} I_{g} \text { (i) (5) }
$$

式中: $I_{G}(i)$ 一一第 $i$ 个基本事件的临界 重要度系数。

\subsection{2 各基本事件临界重要度系数计算}

把表 2 中的基本事件概率值和基本事 件概率重要度系数代入 (5) 式, $I_{G}(1) \sim$ $I_{G}(19)$ 的结果如下:

$$
I_{G}(1)=\frac{q_{l}}{g} I_{g}(1)=\frac{0.1}{0.3205363}
$$

$\times 0.05151996 \approx 0.01607305$

同理: $I_{G}(2) \approx 0.01607305$; $I_{G}(3)=I_{G}(13) \approx 0.02183423 ; I_{G}(4) \approx 0.33551 ;$ $I_{G}(5) \approx 8.6794482 \times 10^{-4} ; I_{G}(6) \approx 4.2698 \times 10^{-}$ ${ }^{3} ; \quad I_{G}(7)=I_{G}(8)=I_{G}(16)=I_{G}(18) \approx 0.0592641 ;$ $I_{G}(9) \approx 1.52757 \times 10^{-3} ; \quad I_{G}(10) \approx 0.590491 ;$ $I_{G}(11) \approx 0.33627788 ; \quad I_{G}(12) \approx 8.647145 \times 10^{-4}$; $I_{G}(14) \approx 6.580609 \times 10^{-3}$; $I_{G}(15) \approx 2.930796 \times 10^{-4}$; $I_{G}(17) \approx 5.387646 \times 10^{-4}$; $I_{G}(19) \approx 4.3136826 \times 10^{-4}$ 。

各基本事件临界重要度系数的排序为: $I_{G}(10)>I_{G}(11)>I_{G}(4)>I_{G}(7)=I_{G}(8)=$

$$
I_{G}(16)=I_{G}(18)>I_{G}(3)=I_{G}(13)>I_{G}(1)=
$$

$I_{G}(2)>I_{G}(14)>I_{G}(6)>I_{G}(9)>I_{G}(5)>I_{G}(12)>$ $I_{G}(17)>I_{G}(19)>I_{G}(15)$ 。

\section{4. 结果分析}

根据结构重要度系数排序, X9、X10 位 居第一位, 在事故树的结构中处于较重要 的地位, 对顶上事件的发生影响因素最大。 从基本事件概率重要度排序得知, X10 的概 率重要度最大, 首先要降低 $X 10$ 发生的概 率。其次 $X 7 、 X 8 、 X 16 、 X 18$ 概率重要度次 之。降低以上基本事件发生的概率, 就能 有效地降低顶上事件发生的概率。根据临 界重要度系数排序分析, $X 10$ 的临界重要度 最大, 必须严格控制 X10, 才能有效地避免 顶上事件发生的可能性。其次还要控制和 解决 $X 11 、 X 4 、 X 7 、 X 8 、 X 16 、 X 18$ 等基本 事件, 也能避免顶上事件的发生。

通过对三个重要度排序的分析得出结论, 儿童攀爬扶手带、对儿童安全教育不足、 未设㞧前阻挡装置、未设坠后防护装置、 无人监护以及家长监护不到位等, 都是造 成儿童意外芏落事故的重要原因。

\section{5. 结论}

事故风险是事故可能性与事故后果严重 性的乘积 ${ }^{[18]}$ 。首先, 减低引起坠落事故发 生可能性的因素, 是自动扶梯坠落风险防 范的首要任务。因此, 对自动扶梯相邻区 域和与建筑物间的危险因素进行风险识别, 加强对施工现场的前期勘验工作, 并对自 
动扶梯的布置环境进行隐患排查, 是提高 自动扶梯相邻区域本质安全的基础工作。

管理部门应引导对自动扶梯相邻区域危 险因素的评估研究, 适时出台相应的自动 扶梯相邻区域防芏落安全防护规定等地方 性法规、规范, 以指导应对和防范芏落风 险工作的实施。

其次, 利用各种宣传手段对家长及儿童 进行适宜的安全乘梯教育, 让其形成自我 约束的安全意识。消除儿童及家长对乘用 自动扶梯危险性认识的盲区, 培养儿童及 家长遵循安全乘梯的良好习惯。

强化使用单位的主体责任, 完善隐患排 查治理责任制度。利用多种监控手段, 实 施现场有效监管, 及时制止乘客不当行为。 维护城市公共安全, 提高公共交通风险防 范能力。

\section{Acknowledgements}

This work was financially supported by the Henan Province Science and Technology Research Project (No.182102210171) and the Henan Provincial Bureau of Quality and Technology Supervision Science and Technology Project (No.2017zj66).

\section{致谢}

本研究得到了河南省科技攻关计划项目 (182102210171) 和河南省质量技术监督 局科技计划项目 (2017zj66) 的资助。

\section{参考文献}

[1] 李耿, 基于安全保障的公共交通型自 动扶梯人机分析与研究, 西安理工大学 硕士学位论文, 2015.

[2] Bardyshev O, Popov V, Druzhinin P, et al. Expert review of metro escalators safety, Transportation Research Procedia, 7(20): 31-35,2017.

[3] 蔡志明, 邵辉, 基于系统动力学方法的 乘坐自动扶梯风险概率研究, 安全与环 境工程, 24 (2) : 141-147, 2017.

[4] Zhang M, Kecojevic V, Komljenovic D. Investigation of haul truck-related fatal accidents in surface mining using fault tree analysis, Safety Science,65(6):106117, 2014.

[5] Jiang W, Qu F, Zhang L. Quantitative
Identification and analysis on hazard sources of roof fall accident in coal mine, Procedia Engineering,45(3):83-88, 2012.

[6] Liu P, Yang L, Gao Z, et al, Fault tree analysis combined with quantitative analysis for high-speed railway accidents, Safety Science,53(79):344-357,2015.

[7] 郭豪收, 张建设, 建筑施工高处坠落伤 亡的事故树安全研究, 山西建 筑, 3 (19) : 197-198, 2007.

[8] 李伯勋, 建筑施工高处芏落事故致因敏 感性分析, 建筑安全, 29 (2):20-22, 2014.

[9] 张玉琪, 机电产品安全性预警模式研究, 沈阳航空航天大学硕士学位论文, 2018.

[10] 机械工业部生产管理局, 事故树分析与 应用, 机械工业出版社, 北京, 1986.

[11] 张明轩, 朱月娇, 翟玉杰, 等, 建筑工程 高处芏落事故的故障树分析方法研究, 煤炭工程, 40 (2) : 112-114, 2008 .

[12] 冯志宏, 建筑施工临边、洞口芏落的事 故树分析及预防对策, 建筑安全, 2010, $25(5): 8-9$

[13] 张乃禄, 刘灿, 安全评价技术, 电子科技 大学出版社, 西安, 2007.

[14] 宁勇, “安防 A 类缺陷 (安全性缺陷) 是 一个也不能有的”, $A \& S$ : 安全\&自动化, $11(70): 73-73,2004$

[15] 陈全, 系统安全工程, 天津科学技术出 版社, 天津, 2010.

[16] 禁运晓, 系统安全工程, 化学工业出版 社, 2009.

[17] 朱继洲, 故障树原理和应用, 西安交通 大学出版社, 西安, 1989.

[18] Xu X, Liu X, Ge G, et al. A method for assessing risk rating of natural gas pipeline based on accident statistics, Journal of Risk Analysis and Crisis Response, 2(1):61-68,2012. 